S. Cazabon

Department of Ophthalmology

St James's University Hospital

Beckett Street

Leeds LS9 7TF, UK

Tel: +44 (0)1132433144

e-mail: jcazabon@hotmail.com

Sir,

\section{Alkali injury complicated by choroidal neovascularisation}

Choroidal neovascularisation occurs in association with age-related macular degeneration, myopia, angioid streaks, ocular histoplasmosis and conditions associated with disruption of Bruch's membrane. It can occur without any associated ocular abnormality. Severe central visual loss occurs due to foveal involvement and macular scarring. The pathogenesis of choroidal neovascularisation is poorly understood. We report a case of an alkali injury which was complicated by the development of choroidal neovascularisation.

\section{Case report}

A 47-year-old Caucasian man presented to the Accident and Emergency department of the Birmingham and Midland Eye Centre. He had accidentally sprayed bleach to both eyes 3 hours previously. He had performed ocular irrigation immediately following the injury. He had previously had excellent visual acuity and was a low myope; his refraction was -2.50 DS in the right eye and -1.75 DS in the left eye. There was no history of previous ocular trauma. He was otherwise healthy. He had no history of foreign travel. There was no family history of ocular disease.

Immediate ocular irrigation was performed on arrival. The $\mathrm{pH}$ measured 8.00 in both eyes. Ocular examination revealed best corrected visual acuity was hand motions (HM) in the right eye and 6/18 in the left eye. Slit-lamp examination of the right eye revealed there was extensive epithelial loss affecting the cornea and conjunctiva, localised corneal oedema and limbal ischaemia extending for $240^{\circ}$, manifest by vascular occlusion. In the left eye there was mild corneal and conjunctival epithelial loss but no evidence of limbal ischaemia. Few fundal details could be discerned due to the anterior segment injuries. He was admitted for intensive treatment with topical corticosteroids, antibiotics, mydriatics, topical and systemic ascorbic acid.

Over the following 4 weeks, his visual acuity in the right eye failed to improve beyond $\mathrm{HM}$ and improved to $6 / 6$ in the left eye. The anterior segment injuries healed with the exception of symblepharon formation in the upper and lower fornices of the right eye (Fig. 1). Fundoscopy revealed a neurosensory detachment overlying the fovea, with retinal pigment epithelial changes and a grey subretinal lesion (Fig. 2a). There was no evidence of peripheral chorioretinal scarring, no

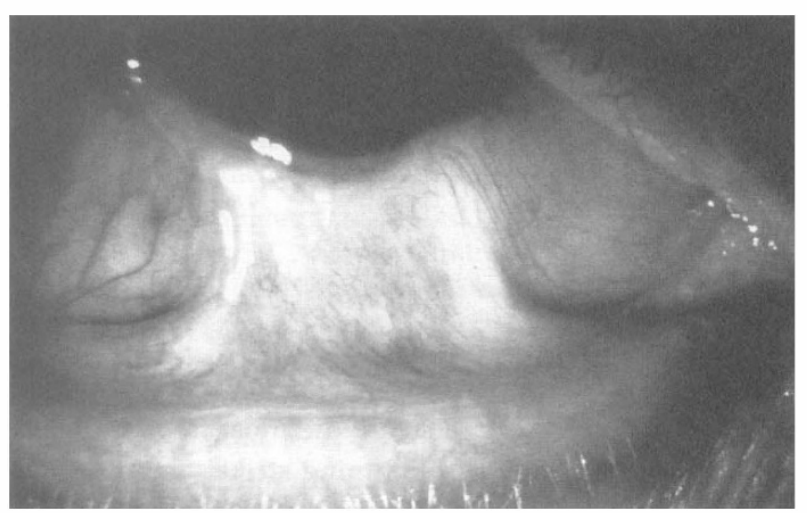

Fig. 1. Slit-lamp photograph of the right eye demonstrating conjunctival scarring and symblepharon formation inferiorly.

histoplasmosis spots and no peripapillary chorioretinal atrophy. Fluorescein angiography confirmed the presence of subfoveal classic choroidal neovascularisation (Fig. 2b, c). Due to the subfoveal location, it was judged that laser photocoagulation would not be of any benefit.

His acuity in the right eye has remained unchanged and he uses topical ocular lubricants to the right eye. His left eye is comfortable and normal.

\section{Comment}

Choroidal neovascularisation is a devastating cause of central visual loss. Ninety per cent of all patients with severe visual loss due to age-related macular degeneration have choroidal neovascularisation. The pathogenesis of choroidal neovascularisation is, however, poorly understood. Alteration to Bruch's membrane plays a key role in the development of choroidal neovascularisation. ${ }^{1}$

Multiple factors are required to induce choroidal neovascularisation. Factors are needed to cause degradation of the extracellular matrix and to stimulate growth of endothelial cells into blood vessels. Angiogenic factors necessary for the development of neovascularisation include vascular endothelial growth factor (VEGF) and fibroblast growth factor (FGF). ${ }^{2}$

The retinal pigment epithelium has been shown in vivo to produce angiogenic factors such as FGF and VEGF. ${ }^{3}$ Retinal pigment epithelium can be activated by a number of stimuli including hypoxia and laser photocoagulation. It has been shown that laser photocoagulation (associated with the development of choroidal neovascularisation) causes release of VEGF and FGF. ${ }^{4}$ It is not known why some eyes which undergo laser photocoagulation develop choroidal neovascularisation and others do not. Ischaemia may play a role, altering the balance between angiogenesis factors and angiogenesis inhibitors.

We report a patient who developed choroidal neovascularisation in association with an alkali injury. Alkali injuries cause ocular ischaemia. We believe that choroidal neovascularisation developed as a result of the 


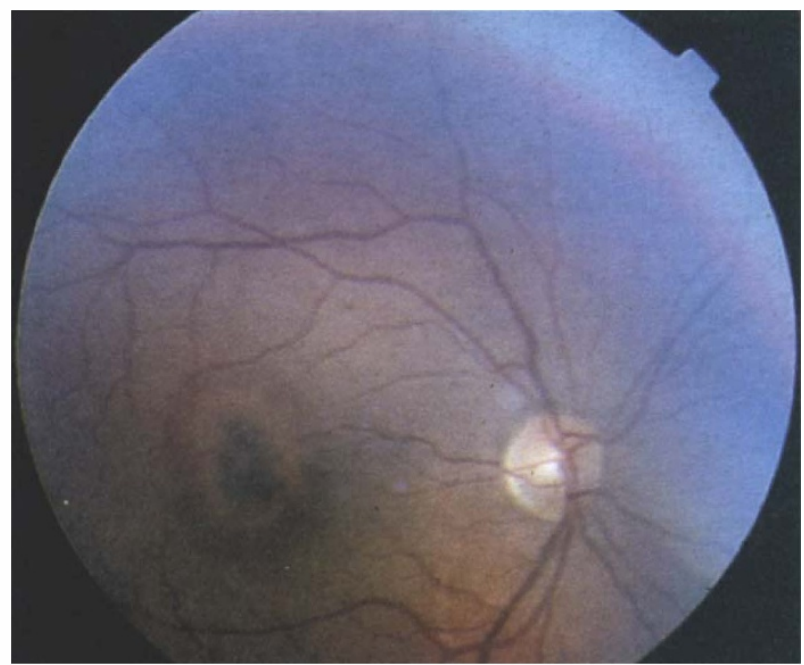

(a)

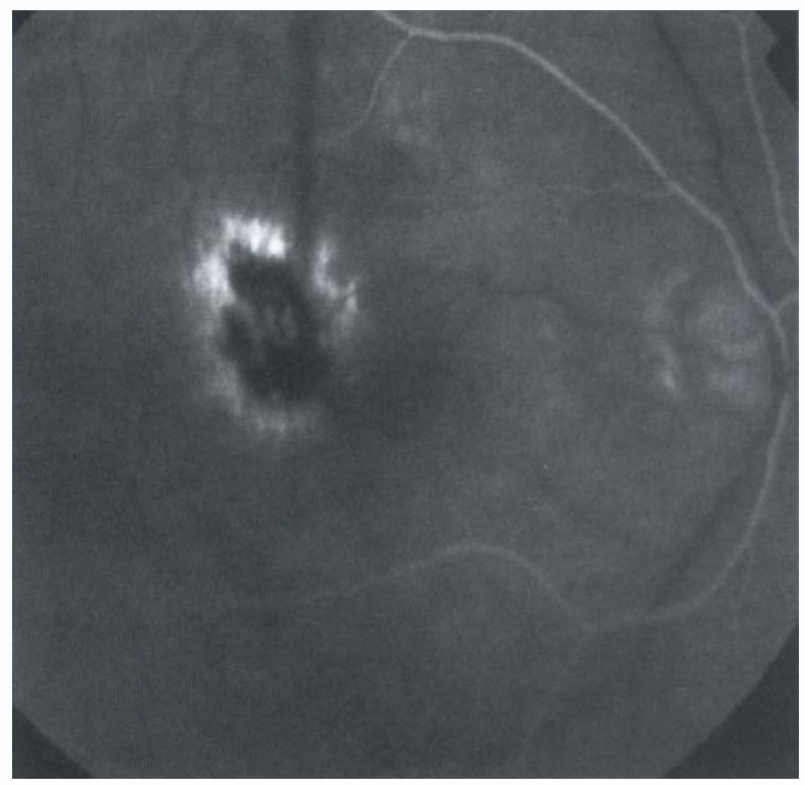

(b)

ocular injury and was possibly precipitated by the associated ocular ischaemia. Ischaemia may play a role in the development of choroidal neovascularisation.

\section{References}

1. Sarks JP, Sarks SH, Killingsworth MC. Morphology of early choroidal neovascularisation in age-related macular degeneration: correlation with activity. Eye 1997;11:515-22.

2. Amin R, Puklin JE, Frank RN. Growth factor localisation in choroidal neovascular membranes of age-related macular degeneration. Invest Ophthalmol Vis Sci 1994;35:3178-88.

3. Frank RN, Amin RH, Eliott D, et al. Basic fibroblast growth factor and vascular endothelial growth factor are present in epiretinal and choroidal neovascular membranes. Am J Ophthalmol 1996;122:393-403.

4. Zhang NL, Samadani EE, Frank RN. Mitogenesis and retinal pigment epithelial cell antigen expression in the rat after krypton laser photocoagulation. Invest Ophthalmol Vis Sci 1993;34:2412-24.

\section{S. Al-Husainy}

P. Stavrou

M.W. Hope-Ross

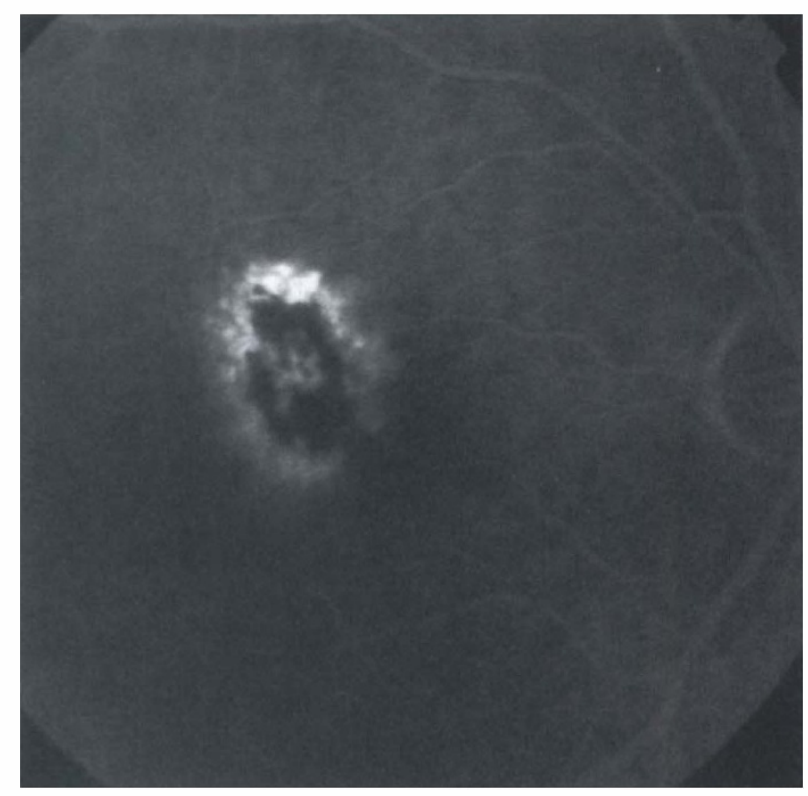

(c)

Fig. 2. (a) Fundus photograph of the right eye demonstrates a grey subretinal lesion beneath the fovea with surrounding retinal pigment epithelial atrophy. (b) Fluorescein angiogram of the right eye in the early phase (20.8 s) shows early lacy hyperfluorescence, representing an area of classic subfoveal choroidal neovascularisation. (c) The latephase angiogram (3.06 $\mathrm{min})$ reveals progressive leakage and a surrounding area of retinal pigement epithelial atrophy.

Birmingham and Midland Eye Centre

Birmingham, UK

Miss M.W. Hope-Ross, MRCPI, FRCS, FRCOphth

Birmingham and Midland Eye Centre

City Hospital NHS Trust

Dudley Road

Birmingham B18 7QU, UK

Tel: +44 (0)121554 3801

e-mail: chell-hope-ross@msn.com

\section{Sir,}

\section{Permanent corneal limbal stem cell dysfunction} following radiotherapy for orbital lymphoma

We present a patient with unilateral stem cell deficiency following the administration of $4600 \mathrm{cGy}$ in 21 fractions for a high-grade malignant $B$ cell lymphoma of the orbit. The stem cell deficiency occurred without acute manifestations of radiation-induced toxicity to the cornea 